OPEN ACCESS

Edited by:

Natarajaseenivasan Kalimuthusamy,

Bharathidasan University, India

Reviewed by:

Muthu Prasad,

Bharathidasan University, India

Erin C. Garcia,

University of Kentucky, United States Kanagavel Murugesan,

Stanford University, United States

*Correspondence:

Yanguang Cong

ygcong@hotmail.com

Weifeng $\mathrm{He}$

heweifeng7412@aliyun.com

Specialty section:

This article was submitted to

Bacteria and Host,

a section of the journal

Frontiers in Cellular

and Infection Microbiology

Received: 30 July 2020

Accepted: 11 December 2020

Published: 27 January 2021

Citation:

Wang J, Xiong K, Pan Q, He W and Cong Y (2021) Application of TonB-

Dependent Transporters in

Vaccine Development of

Gram-Negative Bacteria.

Front. Cell. Infect. Microbiol. 10:589115.

doi: 10.3389/fcimb.2020.589115

\section{Application of TonB-Dependent Transporters in Vaccine Development of Gram-Negative Bacteria}

\author{
Jia Wang ${ }^{1}$, Kun Xiong ${ }^{2}$, Qu Pan ${ }^{3}$, Weifeng $\mathrm{He}^{4 *}$ and Yanguang Cong ${ }^{1,5 *}$ \\ ${ }^{1}$ Department of Clinical Laboratory, Traditional Medicine Hospital Affiliated to Southwest Medical University, Luzhou, China, \\ 2 Department of Cold Environmental Medicine, Institute of High Altitude Military Medicine, Army Medical University, \\ Chongqiong, China, ${ }^{3}$ Department of Microbiology, Chengdu Medical College, Chengdu, China, ${ }^{4}$ Department of Burn, \\ Southwest Hospital, Army Medical University, Chongqing, China, ${ }^{5}$ Precision Medicine Center, Traditional Medicine Hospital \\ Affiliated to Southwest Medical University, Luzhou, China
}

Multiple scarce nutrients, such as iron and nickel, are essential for bacterial growth. Gramnegative bacteria secrete chelators to bind these nutrients from the environment competitively. The transport of the resulting complexes into bacterial cells is mediated by TonB-dependent transporters (TBDTs) located at the outer membrane in Gramnegative bacteria. The characteristics of TBDTs, including surface exposure, protective immunogenicity, wide distribution, inducible expression in vivo, and essential roles in pathogenicity, make them excellent candidates for vaccine development. The possible application of a large number of TBDTs in immune control of the corresponding pathogens has been recently investigated. This paper summarizes the latest progresses and current major issues in the application.

Keywords: TonB-dependent transporter, vaccine, infection, Gram-negative bacteria, immune

\section{INTRODUCTION}

The peptidoglycan of Gram-negative bacteria is encompassed with an outer membrane layer, which serves as a selective permeation barrier and protects bacterial cells from the damage of harmful compounds in the environment. Small hydrophilic nutrients of $<600$ daltons can cross the outer membrane into the periplasmic space via porins in a passive diffusion manner (Nikaido, 2003; Schalk et al., 2012). However, some essential nutrients with large molecular weight or as components of large compounds, which are substantially large to pass through the porin channels, are transported by utilizing TonB-dependent transporters (TBDTs, or TonB-dependent receptors) in the outer membrane (Krewulak and Vogel, 2011).

Most characterized TBDTs are involved in iron uptake (Stork et al., 2013). In vertebrate hosts, iron is sequestered into host proteins, including hemoglobin, myoglobin, transferrin, and ferritin. The iron sequestration is an important defense mechanism that limits the growth of invading pathogens, a strategy known as "nutritional immunity" (Hennigar and McClung, 2016). Pathogens secrete siderophores to bind and chelate iron from iron-containing proteins to circumvent nutritional immunity. Siderophore-iron complexes are subsequently transported into the periplasmic space via TBDTs in an energy-requiring process (Hennigar and McClung, 2016). 
TBDTs are excellent candidates for vaccine development due to their critical roles in bacterial virulence and their surface exposure feature. Thus far, numerous TBDTs have been demonstrated to have vaccine potential against various pathogens. In the present paper, the latest advancements in the application of TBDTs in the vaccine development of Gramnegative bacteria are reviewed, and their future in vaccine application is explored.

\section{STRUCTURE AND FUNCTION OF TBDT}

All TBDTs are structurally similar (Noinaj et al., 2010). The structure of TBDT comprises two domains: a 22 -stranded $\beta$ barrel spanning the outer membrane and a globular plug domain folded into the barrel interior. The $\beta$-barrel domain forms a transport channel for the substrate. The $\beta$-strands are connected sequentially with long loops on the extracellular side of the barrel and short turns on the periplasmic side (Figure 1). The extracellular loops are required for substrate binding and transport through the TBDT protein (Pienko and Trylska, 2020). The plug domain contains a consensus sequence, TonBbox, at the N terminus (Noinaj et al., 2010; Krewulak and Vogel, 2011). The plug domain binds the substrate at the extracellular side of the outer membrane, and its periplasmic region (particularly, the TonB-box) interacts with the TonB-ExbBExbD complex to tap energy derived from the proton motive force across the inner membrane, which is required for the substrate transport. The substrate in the periplasmic space is subsequently transferred into the cytoplasm through an $\mathrm{ABC}$ transporter in the inner membrane (Figure 2) (Noinaj et al., 2010; Krewulak and Vogel, 2011; Gresock and Postle, 2017). Siderophores are the most common transport substrates of TBDTs. However, recent studies have revealed the diversity of TBDT substrates, including vitamin B12, nickel chelates, zinc chelates, carbohydrates, and enzymes (Schauer et al., 2008).

\section{CHARACTERISTICS OF TBDTS MAKE THEM EXCELLENT CANDIDATES FOR THE VACCINE DEVELOPMENT OF GRAM- NEGATIVE BACTERIA}

An ideal target for the vaccine development should have the following features: (i) surface exposure, (ii) wide distribution in pathogenic strains, (iii) capable of eliciting protective immune responses, (iv) inducible expression during infection, and (v) plays a critical role in pathogenesis (Wieser et al., 2010).

The characteristics of TBDTs meet most standards of the ideal target for the vaccine development. As outer membrane proteins, TBDTs have many surface-exposed epitopes, which are targets of the host immune system (Solis and Cordwell, 2011; Zeng et al., 2017). For example, the extracellular loops that connect the $\beta$-strands of TbpA, a TBDT essential for the uptake of transferrin-iron in Neisseria gonorrhoeae, are surface-exposed and immunogenic. The specific antibodies against these loops can prevent the ligand binding capabilities of TbpA, therefore, inhibit bacterial growth in vivo (Boulton et al., 2000; Cornelissen et al., 2000; Masri and Cornelissen, 2002).

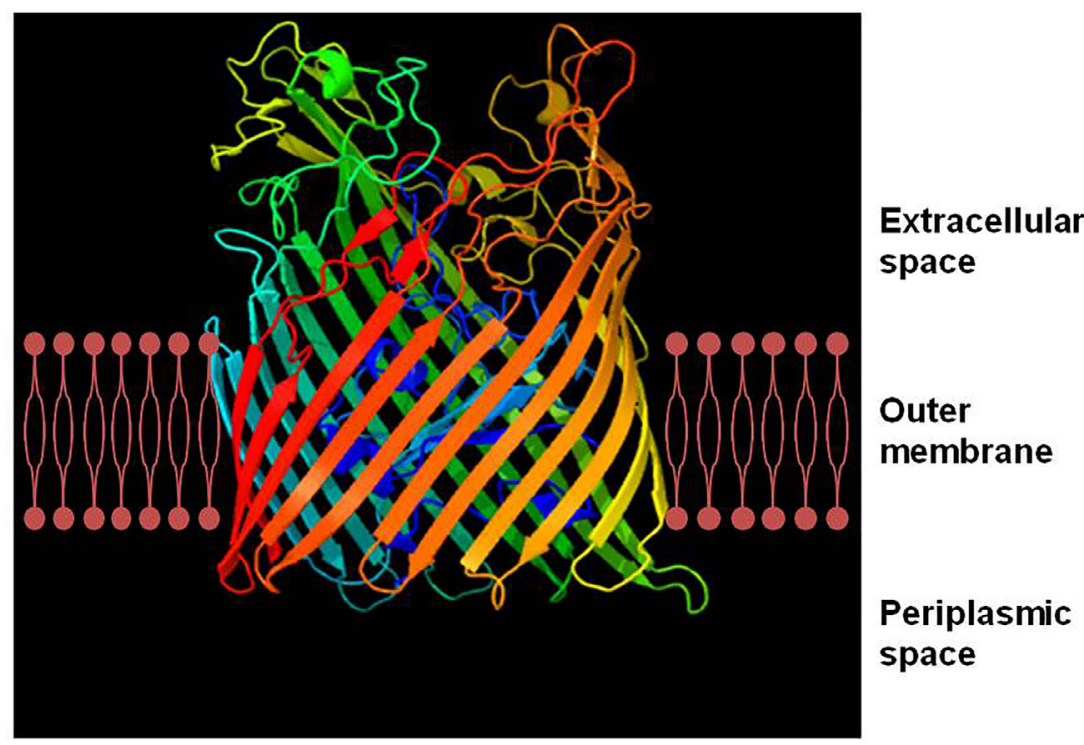

FIGURE 1 | Ribbon diagram of FepA, a TonB-dependent transporter (TBDT) from Escherichia coli. TonB-dependent transporters possess two domains, a 22stranded anti-parallel $\beta$-barrel spanning the outer membrane and an N-terminal globular plug domain that resides within the barrel. The $\beta$-strands of the barrel are connected on the extracellular side by long flexible loops and on the periplasmic side by short turns. The ribbon diagram of FepA was generated with the Phyre2 software (Kelley et al., 2015). 


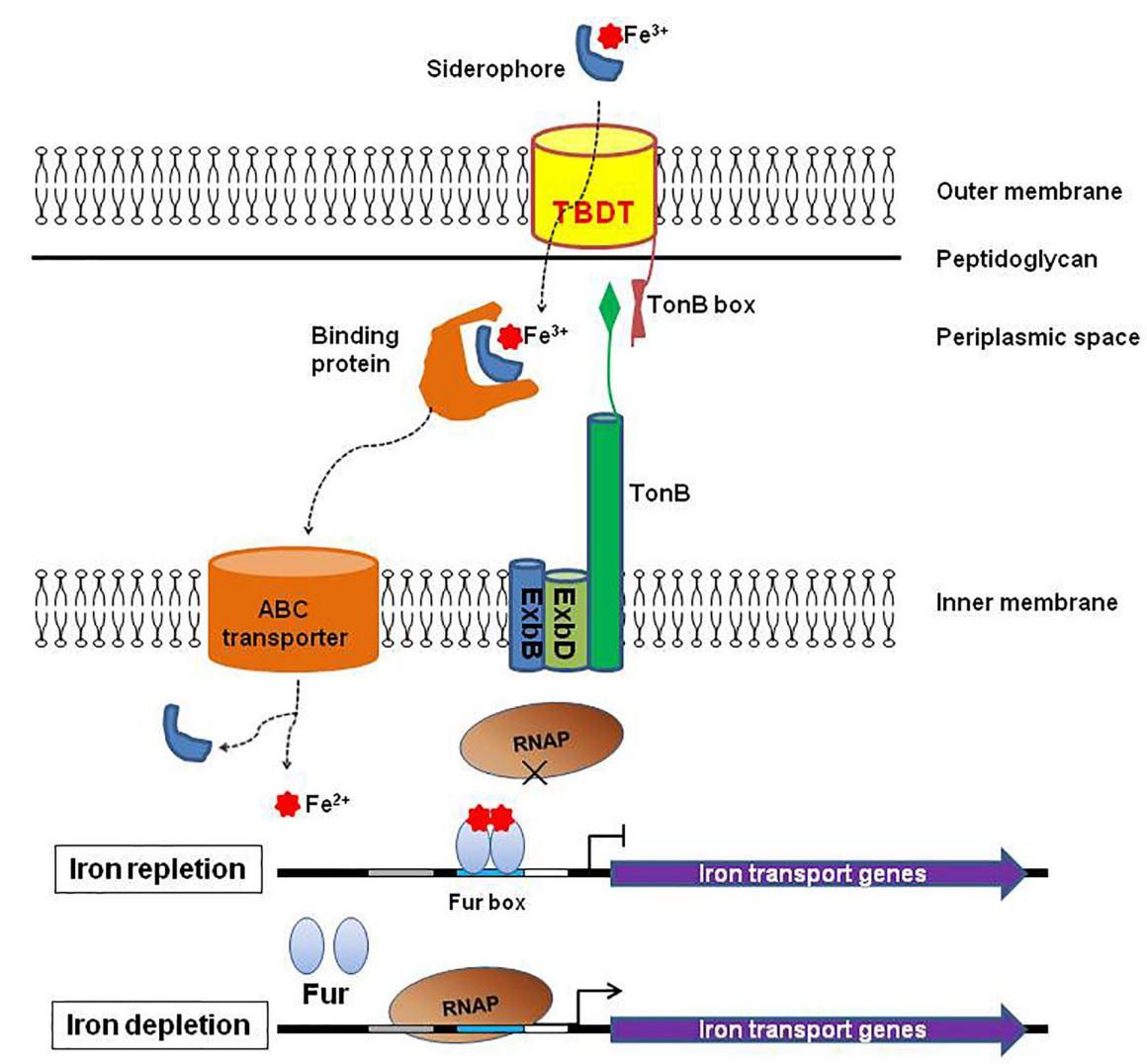

FIGURE 2 | Diagram of substrate transport via TonB-dependent transporter (TBDT). Siderophore secreted by Gram-negative bacteria chelates ferric ion (Fe ${ }^{3+}$ ) from $\mathrm{Fe}^{3+}$-containing compounds in the extracellular environment. TonB-dependent transporter (TBDT) then mediates transport of ferric siderophore through the outer membrane into the periplasmic space. This process is driven by energy from an inner membrane protein complex, TonB-ExbB-ExbD. In the periplasm, the ferric siderophore is taken up by a binding protein and delivered through the inner membrane by an ABC-type transport complex. There the ferric siderophore is hydrolyzed, and then reduced to ferrous ion $\left(\mathrm{Fe}^{2+}\right)$ by enzymes to supply iron for cell metabolism. Expression of iron-transport genes (including TBDT-encoding genes) is under the strict regulation of the ferric uptake regulator (Fur). Fur binds the Fur box sequence within the promoter region of the target genes using excess $\mathrm{Fe}^{2+}$ as a cofactor, and blocks the access of RNA polymerase leading to the repression of the target genes. However, when Fe ${ }^{2+}$ is depleted, Fur cannot bind the Fur box resulting in depression of the target genes.

Moreover, the specific antibodies can bind to the TBDT epitopes of Acinetobacter baumannii and form immune complexes, which can be recognized by phagocytes and facilitate bacterial elimination (Goel and Kapil, 2001).

Due to the important roles of substrates in bacterial survival in the host, TBDTs are required for the pathogenicity of various pathogens, including Salmonella enterica (Kingsley et al., 1999; Williams et al., 2006; Hu et al., 2009; Xiong et al., 2012), Francisella tularensis (Sen et al., 2010), Pseudomonas fluorescens (Sun and Sun, 2015), Haemophilus ducreyi (Leduc et al., 2008), Riemerella anatipestifer (Lu et al., 2013), Proteus mirabilis (Nielubowicz et al., 2008), Pseudomonas aeruginosa (Mastropasqua et al., 2017), Neisseria meningitidis (Kumar et al., 2012), et al. For example, The catecholate siderophores have a strong affinity for iron (Wandersman and Delepelaire, 2004), and play a key role in the iron uptake and in vivo growth of Salmonella enterica (Williams et al., 2006; Wellawa et al., 2020). Transport of these catecholate siderophores is dependent on three TBDTs, IroN, FepA, and CirA. Therefore, deficiency of these transporters of catecholate siderophores led to significant virulence reduction in $S$. Typhimurium (Wellawa et al., 2020).

The TBDTs proteins are highly regulated at transcriptional and posttranscriptional levels; and the virulence-associated TBDTs are generally induced in vivo (Hagan and Mobley, 2007; Noinaj et al., 2010). For example, the ferric uptake regulator (Fur) plays a key role in the regulation of TBDTs for siderophore (Sun and Sun, 2015; Becerra et al., 2020). In the presence of iron, Fur binds a specific 19 bp DNA sequence, called the "Fur box", using $\mathrm{Fe}^{2+}$ as a cofactor within the promoter region of the regulated genes. The binding of Fur prevents the access of RNA polymerase leading to the repression of the downstream genes. However, under iron limiting conditions (for example, in vivo), Fur cannot bind the sequence of the Fur box resulting in derepression of genes that encode siderophore transporters (including TBDTs), proteins involved in siderophore biosynthesis and iron metabolism (Figure 2) (Lee and Helmann, 2007; Noinaj et al., 2010). In addition to Fur, 
multiple regulatory factors including small RNAs, ChvR (Frohlich et al., 2018), OmrA and OmrB (Guillier and Gottesman, 2008), string response regulators (P) ppGpp and DksA (Zhang et al., 2019), participate in the regulation of TBDTs for siderophore.

\section{APPLICATION OF TBDTS IN THE VACCINE DEVELOPMENT OF GRAM- NEGATIVE BACTERIA}

\section{Attenuated Vaccine}

Because many of the substrates transported by TBDTs are essential for the survival and pathogenicity of pathogens in vivo, mutation of the encoding genes of these TBDTs will lead to significant decrease of bacterial virulence. Therefore, some TDBT-encoding genes are suitable targets for the construction of attenuated vaccine strains (Table $\mathbf{1}$ ).

\section{Salmonella enterica}

Three TBDTs are available for the transport of catecholate siderophore in Salmonella enterica. Cir transports 2, 3dihydroxybenzoylserine; FepA facilitates the uptake of enterobactin and 2, 3-dihydroxybenzoylserine; IroN is the receptor for salmochelins, enterobactin, and 2, 3dihydroxybenzoylserine. Intragastric and intravenous immunization of the triple deletion mutant elicited significant protective immune responses against the wild-type challenge by either route in the mouse model (Williams et al., 2006).

FoxA mediates the transport of ferrioxamines across the outer membrane. A foxA mutation substantially reduced bacterial colonization in rabbit ileal loops, resulting in virulence reduction. Furthermore, immunization of the fox $A$ mutant protected mice against the wild-type challenges (Kingsley et al., 1999).

YncD is a putative TBDT of $S$. Typhi, which had been identified as an in vivo-induced antigen probed with sera from Typhoid fever patients (Hu et al., 2009). Nasal inoculation of the yncD-deleted mutant elicited a significant immunoprotection against the lethal wild-type challenge (Xiong et al., 2012). The $y n c D$ gene was also used in the construction of the attenuated vaccine strains of S. Paratyphi A (Xiong et al., 2015; Zhu et al., 2015).

\section{Shigella dysenteriae}

In some cases, TBDT-encoding genes were used as one of the target genes in strain construction. For example, the SC599 vaccine is an attenuated strain of $S$. dysenteriae with the deletion of four genes. The fep gene that encodes a TBDT for enterobactin, was deleted in addition to the mutation of three other virulence genes, $i c s A$ (essential for bacterial spreading), ent (encoding enterobactin synthase), and stxA (encoding the shiga toxin active A subunit) to construct the strain. Clinical trials revealed that the SC599 vaccine was well tolerated. A single oral vaccination elicited significant IgA ASCs and antibody responses (Sadorge et al., 2008; Launay et al., 2009).

\section{Riemerella anatipestifer}

$R$. anatipestifer, which causes acute septicemia and infectious polymastitis in ducks, chickens, geese, and other avian species, is a serious threat to the poultry industry. Liu et al. demonstrated that a putative TonB-dependent iron transporter of $R$. anatipestifer CH-1, namely, B739_1343, played an important role in iron acquisition and bacterial pathogenicity. The deletion of B739_1343 led to a significant decrease in virulence. Intramuscular immunization with the deletion mutant protected $83.33 \%$ of the immunized ducks against intramuscular challenges of the wild-type strain at a high dose of 100-fold $\mathrm{LD}_{50}$ (Liu et al., 2018).

\section{Subunit Vaccine}

TBDTs comprise a large part of the identified proteins with vaccine potential in large-scale screens based on genomics, proteomics, reverse and structural vaccinology. A total of $18 \beta$-barrel transmembrane proteins and 8 outer membrane lipoproteins were identified in an effort to discover novel leptospiral vaccine candidates. Many $\beta$-barrel transmembrane proteins (6/18) are TonB-dependent receptors associated with nutrient transportation (Grassmann et al., 2017). In another effort to determine potential core vaccine targets of $A$. baumannii, two TBDTs, namely FhuE receptor and HMPREF0010_01517, are identified (Hassan et al., 2016). Researchers used a large-scale selection process combined with bioinformatic, genomic, transcriptomic, and proteomic in a screen of vaccine candidate proteins for uropathogenic Escherichia coli. Six suitable vaccine candidates, including ChuA, Hma, Iha, IreA, IroN, and IutA, were screened from the 5379 predicted proteins. All these candidates are TBDTs involved in iron uptake (Alteri et al., 2009). So far many TBDTs from various pathogens had been evaluated their potential as subunit vaccines. The important progresses are summarized in Table 1 and described in the following sections.

\section{Uropathogenic Escherichia coli (UPEC)}

UPEC is among the most prevalent agents of urinary tract infections. Four TBDTs (IreA, Hma, IutA, and FyuA) associated with iron acquisition had been identified in systematic screens. Nasal immunization of each antigen, with cholera toxin as adjuvant, protected experimentally infected mice from colonization of the bladder and/or kidneys by UPEC (Alteri et al., 2009; Serino et al., 2010; Brumbaugh et al., 2013; Habibi et al., 2017). The four antigens can be combined to produce a multi-subunit vaccine (Mobley and Alteri, 2015).

\section{Pseudomonas aeruginosa}

$P$. aeruginosa is one of the leading opportunistic pathogens responsible for acute and chronic respiratory tract infections with high morbidity and mortality. An in vivo-induced ferripyoverdine transporter, FpvA, is essential for virulence of $P$. aeruginosa. A subunit vaccine comprising the extracellular peptides of FpvA conjugated to keyhole limpet hemocyanin $(\mathrm{KLH})$ was produced to evaluate its immunoprotective efficacy in a murine model. Nasal vaccination of FpvA-KLH elicited IgG and $\operatorname{IgM}$ antibodies in sera and $\operatorname{IgA}$ antibodies in lung supernatant. $\mathrm{CD} 11 \mathrm{~b}^{+}$dendritic cells and memory $\mathrm{CD}^{+} \mathrm{T}$ cells 
TABLE 1 | Application of TonB-dependent transporters (TBDTs) in vaccine development of Gram-negative bacteria.

\begin{tabular}{|c|c|}
\hline Vaccine candidates & Results \\
\hline \multicolumn{2}{|l|}{ Attenuated vaccine } \\
\hline $\begin{array}{l}\text { Salmonella enterica } \\
\text { the fepA iroN cir triple } \\
\text { mutant }\end{array}$ & $\begin{array}{l}\text { The mutant was significantly attenuated in a mouse model. No immunized mice were killed by the i.g. or i.v. } \\
\text { challenges at lethal doses. }\end{array}$ \\
\hline the foxA mutant & $\begin{array}{l}\text { The } L D_{50} \text { of the mutant }\left(>10^{9} \mathrm{CFU} \text { i.g.; }>10^{4} \mathrm{CFU} \text { i.v.) were significantly higher than those of the wild-type }\right. \\
\left(10^{5} \mathrm{CFU} \text { i.g.; }<10 \mathrm{CFU} \text { i.v.). Immunization of the mutant significantly protected the immunized mice from the }\right. \\
\text { intragastric challenge of the wild-type. }\end{array}$ \\
\hline$\Delta y n c D$ & $\begin{array}{l}\text { The } \Delta y n c D \text { mutant is } 1000 \text { times less virulent than the wild-type in the mouse mucin model. Intranasal } \\
\text { inoculation of } \Delta y n c D \text { showed a significant immunoprotection against the lethal intraperitoneal challenge of the } \\
\text { wild-type. }\end{array}$ \\
\hline $\begin{array}{l}\text { Shigella dysenteriae } \\
\Delta i c s A \Delta \text { ent } \Delta \text { fep } \Delta \text { stxA }\end{array}$ & $\begin{array}{l}\text { Clinical trials showed that the vaccine was well tolerated (the maximum tolerable dose }>10^{8} \mathrm{CFU} \text { ). A single } \\
\text { oral vaccination induced a significant circulating IgA ASC and serum antibody response. }\end{array}$ \\
\hline \multicolumn{2}{|l|}{ Subunit vaccine } \\
\hline $\begin{array}{l}\text { Uropathogenic } \\
\text { Escherichia coli } \\
\text { IreA, Hma, lutA, FyuA }\end{array}$ & $\begin{array}{l}\text { Intranasal immunization of mice with the four TBDTs stimulates a protective systemic and mucosal immune } \\
\text { responses that correlate with significant reductions in bladder and/or kidney bacterial load and significant } \\
\text { protection from experimental infection. }\end{array}$ \\
\hline $\begin{array}{l}\text { Pseudomonas } \\
\text { aeruginosa }\end{array}$ & $\begin{array}{l}\text { Intranasal peptide-based FpvA-KLH conjugate vaccine of mice elicited production of IgG and IgM antibodies } \\
\text { in sera, IgA antibodies in lung supernatant and antigen-specific IL-17. FpvA-KLH immunization significantly }\end{array}$ \\
\hline FpvA & $\begin{array}{l}\text { reduced bacterial burden and pulmonary edema, protected mice from acute murine pneumonia of } P \text {. } \\
\text { aeruginosa. }\end{array}$ \\
\hline $\begin{array}{l}\text { Neisseria meningitidis } \\
\text { TbpA }\end{array}$ & $\begin{array}{l}\text { Subcutaneous inoculation of mice with recombinant TbpA conferred significant protection ( } 100 \% \text { at the } \\
\text { challenge dose of } 2 \times 10^{7} \mathrm{CFU;} 85 \% \text { at the challenge dose of } 2 \times 10^{8} \mathrm{CFU} \text { ) against the lethal challenges of } N \text {. } \\
\text { meningitidis. }\end{array}$ \\
\hline
\end{tabular}

FetA (FrpB, HxuC)

ZnuD

Acinetobacter

baumannii

BauA, BfnH

Extraintestinal

pathogenic Escherichia

coli

lutA, IroN

FyuA, IroN, lutA, ChuA

Campylobacter jejuni

CfrA

Salmonella enterica

IroN

Neisseria gonorrhoeae

TbpA

Klebsiella pneumonia

Kleb-SRP

Aeromonas hydrophila

$\mathrm{Tdr}$

Pseudomonas

fluorescens

TdrA

TfeR
ZnuD can elicit bactericidal antibodies upon immunization in mice.

Subcutaneous immunization of mice with BauA and $\mathrm{BfnH}$ conferred survival rates of $45 \%$ and $40 \%$, respectively, against the lethal challenge of $A$. baumannii. Passive immunization resulted in approximately $50 \%$ of survival rate in the groups receiving anti-BfnH or a combination of anti-BfnH and anti-BauA. Immunization of mice with recombinant lutA or IroN elicited high liters of total IgG antibody of IgG1/lgG2a isotypes at day 40 post-immunization, leading to protection against the lethal and non-lethal sepsis challenges.

Nasal administration of two artificial chimeric polypeptides comprising the immunogenic epitopes of FyuA, responses, and conferred protection with a significant reduction of bacterial load in a mouse model of ExPEC peritonitis.

CfrA-specific lgG blocked the ligand binding site of CfrA and consequently inhibited enterobactin-mediated growth promotion under iron-restricted conditions. The inhibitory effect was dose dependent.

Intramuscular immunization of chicken with IroN elicited a survival rate of $90 \%$ against the intravenous challenge of $S$. Enteritidis relative to the $20 \%$ survival rate of the control group.

Vaccine formulations comprising epitopes of TbpAB and inactivated cholera toxin induced potentially protective antibodies.

Administration of cows with the vaccine before calving significantly decreased the risk of Klebsiella and total coliform mastitis by $76.9 \%$ and $47.5 \%$ respectively.

Immunization with recombinant Tdr protein emulsified with non-mineral oil adjuvant protected Catfish fingerlings against the challenge of the virulent $A$. hydrophila strain with a relative survival rate of 95.59\%. The bacterial burdens in the liver, spleen, and anterior kidney of the immunized catfish were also markedly reduced.

Immunization with recombinant TdrA protected the immunized flounders against the lethal challenge of $P$. fluorescens with a relative percent of survival of $80.6 \%$.

Immunization of turbot with recombinant TfeR induced high titers of specific serum antibodies, which bound the $P$. fluorescens cells, and greatly reduced bacterial infectivity to fish cells. The relative percent of survival of the TfeR immunization was 50\%. IroN, lutA, ChuA, and UPEC-specific protein UspA stimulated strong humoral, cellular, and mucosal immune

Kleb-SRP was prepared by purifying siderophore receptor and porin proteins from $K$. pneumonia.

\section{References}

(Williams et al., 2006)

(Kingsley et al., 1999)

(Xiong et al., 2012)

(Sadorge et al., 2008; Launay et al., 2009)

(Liu et al., 2018)

(Alteri et al., 2009; Serino et al., 2010; Brumbaugh et al., 2013; Mobley and Alteri, 2015; Habibi et al., 2017)

(Sen-Kilic et al., 2019)

(West et al., 2001)

(Pettersson et al., 1990; Ala'Aldeen et al., 1994; Kortekaas et al., 2006)

(Stork et al., 2010)

(Aghajani et al., 2019)

(Mellata et al., 2016)

(Wieser et al., 2010)

(Zeng et al., 2009)

(Kaneshige et al., 2009)

(Cornelissen, 2008)

(Gorden et al., 2018)

(Abdelhamed et al., 2017)

(Hu et al., 2012)

(Sun and Sun, 2015) 
TABLE 1 | Continued

\begin{tabular}{|c|c|c|}
\hline Vaccine candidates & Results & References \\
\hline $\begin{array}{l}\text { Haemophilus ducreyi } \\
\text { HgbA }\end{array}$ & $\begin{array}{l}\text { Anti-HgbA IgG blocked hemoglobin binding to the HgbA receptor. Immunization with HgbA reduced initial } \\
\text { size and severity of lesion formation and/or increased the speed of healing in a swine model of chancroid } \\
\text { infection. Immunized pigs effectively eliminated pathogens at the inoculation site. Passive immunization also } \\
\text { protected the pigs from the homologous challenge. }\end{array}$ & $\begin{array}{l}\text { (Afonina et al., 2006; Nepluev } \\
\text { et al., 2009; Leduc et al., 2011) }\end{array}$ \\
\hline $\begin{array}{l}\text { Haemophilus parasuis } \\
\text { HxuC }\end{array}$ & $\begin{array}{l}\text { Vaccination of mice with } \mathrm{HxuC} \text { elicited significant humoral immune responses and increased the levels of IL- } \\
2 \text {, IL-4, IFN- } \gamma \text {. The HxuC immunization alleviated the tissue damage levels in the mouse model of } \\
\text { intraperitoneal infection of } H \text {. parasuis and conferred protection against the lethal challenge with a survival } \\
\text { rate of } 87.5 \% \text {. }\end{array}$ & (Wen et al., 2016) \\
\hline FhuA & FhuA was immunogenic and elicited specific antibodies during the course of natural infection. & (del Rio et al., 2006) \\
\hline $\begin{array}{l}\text { Pasteurella multocida } \\
\text { TbpA }\end{array}$ & $\begin{array}{l}\text { Subcutaneous immunization with recombinant TbpA fragments with varied lengths stimulated high levels of } \\
\text { total IgG and subtypes IgG1 and IgG2a in the mice serum. The immunization protected the immunized mice } \\
\text { against lethal challenges with survival rates from } 16.7 \%-50 \% \text {. }\end{array}$ & (Shivachandra et al., 2015) \\
\hline
\end{tabular}

$L D_{50}$, median lethal dose; i.g., intragastrically; i.v., intravenously.

were induced in the lungs of immunized mice. Furthermore, FpvA-KLH immunization significantly reduced bacterial burden and pulmonary edema (Sen-Kilic et al., 2019).

\section{Neisseria meningitidis}

$N$. meningitidis is the etiologic agent of meningococcal meningitis, which is a serious public health issue in developed and developing countries. Neisseria cells steal iron from human iron-binding proteins, such as hemoglobin, transferrin, and lactoferrin, for survival. The immunoprotection of recombinant transferrin binding proteins, TbpA (a TBDT) and $\mathrm{TbpB}$, had been evaluated against meningococcal infection in a mouse model. TbpA was demonstrated to confer protection against the challenge of $N$. meningitidis and had crossprotection against the isolates of serogroup $\mathrm{B}$ and serogroup $\mathrm{C}$ (West et al., 2001; Noinaj et al., 2012; Noinaj et al., 2013).

FetA (also known as FrpB, HxuC) is an outer membrane heme transporter of $N$. meningitidis (Saleem et al., 2012). FetA has been demonstrated to induce bactericidal antibody and regarded as a vaccine component (Pettersson et al., 1990; Ala'Aldeen et al., 1994; Kortekaas et al., 2006). However, the antibody is strain-specific due to the high variations of surfaceexposed fragments (Thompson et al., 2003).

In addition to TonB-dependent iron transporters, an outer membrane zinc transporter, $\mathrm{ZnuD}$, is also required for bacterial survival and plays a critical role in the pathogenicity of $N$. meningitidis. ZnuD, which is highly conserved in the isolates of N. meningitidis, can elicit bactericidal antibodies upon immunization in mice (Stork et al., 2010).

\section{Acinetobacter baumannii}

A. baumannii is an opportunistic pathogen that accounts for serious life-threatening nosocomial infections due to its easy acquisition of antibiotic resistance and its persistence in the environment (Greene et al., 2016). BauA and $\mathrm{BfnH}$ are two TBDTs in A. baumannii that facilitate the uptake of siderophores from the acinetobactin cluster and cluster I, respectively (Eijkelkamp et al., 2011). Both TBDTs were identified as vaccine candidates using an assay of reverse vaccinology $(\mathrm{Ni}$ et al., 2017). The whole proteins of BauA and $\mathrm{BfnH}$ showed partial protective effects in animal experiments. Death rate and bacterial burdens in the main organs of immunized mice were decreased compared with those of the control group. Passive immunization resulted in approximately $50 \%$ of survival rate in the immunized groups of $\mathrm{BfnH}$ or a combination of $\mathrm{BfnH}$ and BauA (Aghajani et al., 2019).

\section{Extraintestinal Pathogenic Escherichia coli (ExPEC)}

ExPEC reside in the intestine as members of normal flora. However, ExPEC cells cause infections when entering extraintestinal sites, such as blood, urinary tract, and meninge, due to their specific virulence factors. ExPEC are the leading cause of community and nosocomial bacterial sepsis with a mortality rate of $30 \%-50 \%$. Salmochelin and aerobactin mediate the iron uptake of ExPEC, which is essential for bacterial virulence (Garenaux et al., 2011). Their corresponding transporters, IroN and IutA, are widely distributed in human isolates of ExPEC (Mellata et al., 2009).

Immunogenicity of IroN was first evaluated by Russo et al. (2003). Subcutaneous inoculation of recombinant IroN induced a significant increase in anti-IroN IgG in immunized mice, leading to a significant reduction of bacterial burdens in the kidneys but not the bladders of the mice challenged with ExPEC. Subcutaneous immunization of mice with recombinant IutA and IroN elicited strong humoral immune responses and protected immunized mice against the lethal and non-lethal sepsis challenges. Passive immunization also reduced bacterial burdens in organs and blood of the tested mice (Mellata et al., 2016).

Wieser et al. in silico predicted immunogenic epitopes of the extracellular loops of the E. coli outer membrane siderophore receptor FyuA, IroN, and IutA, the heme/hemoglobin TonBdependent receptor ChuA, and the uropathogenic E. coli (UPEC)-specific protein UspA, and combined them into two artificial chimeric polypeptides. Nasal administration of the two novel multi-epitope subunit vaccines elicited strong humoral and cellular immune responses, and conferred a high degree of protection in a mouse model of ExPEC peritonitis (Wieser et al., 2010).

\section{Campylobacter jejuni}

Campylobacter is one of the most common bacterial causes of human enteritis in many industrialized countries (Ketley, 1997). 
Zeng et al. found that a ferric enterobactin receptor, CfrA, was dramatically induced under iron-restricted conditions and during infections. The anti-CfrA serum blocked the function of iron uptake and diminished ferric enterobactin-mediated growth promotion under iron-restricted conditions (Zeng et al., 2009).

\section{Salmonella enterica}

Vaccination is one of the efficient means to control the contamination of Salmonella Enteritidis in poultry farming. Kaneshige et al. purified natural IroN from $S$. Typhimurium using affinity chromatography. Intramuscular immunization of chicken with purified natural IroN conferred a survival rate of $90 \%$ against the intravenous challenge of $S$. Enteritidis compared with the $20 \%$ survival rate of the control group (Kaneshige et al., 2009). The data showed that IroN is an important protective antigen against Salmonella infection in chickens.

\section{Neisseria gonorrhoeae}

$N$. gonorrhoeae causes gonorrhea, a global public health issue with an estimated incidence of 62.4 million cases each year. The transferrin iron transport system, $\mathrm{TbpAB}$, is not subject to antigenic variation and is widely distributed in Neisseria isolates. Vaccine formulations comprising epitopes of TbpAB elicited potentially protective antibodies (Cornelissen, 2008).

\section{Klebsiella pneumonia}

Mastitis caused by Klebsiella spp. is an emerging issue in the dairy industry. A K. pneumoniae vaccine (Kleb-SRP) was prepared by purifying siderophore receptor and porin proteins from fermentation cultures of K. pneumonia. Subcutaneous vaccination of cows with Kleb-SRP before calving decreased the risk of Klebsiella and total coliform mastitis by $76.9 \%$ and $47.5 \%$, respectively (Gorden et al., 2018).

\section{Aeromonas hydrophila}

A TonB dependent transporter, Tdr, presents in the virulent strains of $A$. hydrophila but was absent in strains of low virulence. Immunization with $\mathrm{Tdr}$ induced significant antibody responses and protected immunized catfish fingerlings against the challenge of the virulent strain, and the relative survival rate was $95.59 \%$. The bacterial burdens in the organs of immunized catfish were also markedly reduced (Abdelhamed et al., 2017).

\section{Pseudomonas fluorescens}

P. fluorescens is a common pathogen of aquaculture. The purified recombinant TdrA, a TonB-dependent outer membrane transporter, is a promising subunit vaccine. Immunization of recombinant TdrA protected the immunized flounders effectively against the lethal challenge of $P$. fluorescens with a relative percent of survival of $80.6 \%$ (Hu et al., 2012).

A ferric enterobactin transporter of $P$. fluorescens, TfeR, is essential for bacterial virulence in turbot. Inoculation of recombinant $\mathrm{TfeR}$ induces specific serum antibodies and displays significant protection against the challenge of pathogenic strain. The TfeR antibodies bind with bacteria and block their infection (Sun and Sun, 2015).

\section{Haemophilus ducreyi}

$H$. ducreyi is the cause of chancroid. A hemoglobin transporter, $\mathrm{HgbA}$, promotes heme acquisition and is required for the survival and pathogenicity of $H$. ducreyi. Immunization of purified native HgbA prevented the development of chancroid in a swine model. Immunized animals effectively eliminated pathogens at the inoculation site. Anti-HgbA IgG blocks the binding of hemoglobin to HgbA and restricts the capability of $H$. ducreyi to acquire heme/iron and then promotes the elimination of pathogens (Afonina et al., 2006; Nepluev et al., 2009; Leduc et al., 2011).

\section{Haemophilus parasuis}

$H$. parasuis is the etiological agent of Glasser's disease in pigs. The $h x u C$ gene encodes a transporter for heme/hemopexin utilization and is required for bacterial virulence (Morton et al., 2007). Wen et al. found that immunization of HxuC induced strong immune responses and protected immunized mice against the lethal challenge of the virulent strain with $87.5 \%$ survival rate. The antiserum inhibited the bacterial growth of $H$. parasuis (Wen et al., 2016). FhuA is involved in ferric hydroxamate uptake of $H$. influenzae. Animal experiments showed that FhuA was immunogenic and induced specific antibodies during porcine infection (del Rio et al., 2006).

\section{Pasteurella multocida}

Hemorrhagic septicemia is a serious disease of cattle and buffalo mainly caused by the pathogenic serogroups of $P$. multocida (Shivachandra et al., 2015). Transferrin-binding protein A (TbpA) is an iron acquisition TBDT prevalent in P. multocida. Shivachandra et al. evaluated the immunogenicity of recombinant TbpA fragments with varied lengths. Immunization with TbpA fragments induced high levels of IgG, IgG1, and IgG2a in the mice serum and protected the immunized mice against lethal challenges with survival rates ranging from $16.7 \%-50 \%$ (Shivachandra et al., 2015).

\section{ADVANTAGES AND DISADVANTAGES OF TBDTS IN THE VACCINE DEVELOPMENT}

Numerous studies have shown that TBDTs conferred significant immune protection against infection caused by their host bacteria. The characteristics revealed in these studies make TBDTs excellent candidates for the vaccine development (Table 2). However, antigenic variation hinders the application of some TBDTs in the vaccine development (Table 2).

Due to their surface location and key roles in virulence, TBDTs are subjected to high selection pressure, which results in frequent variations in some TBDTs. For example, vaccine efforts of $N$. gonorrhoeae have been hampered by the frequent antigenic variation of most surface structures. N. gonorrhoeae has two variation mechanisms. The first one is homologous recombination, which is employed by the pilin protein (Criss et al., 2005). The second mechanism is the slipped-strand mispairing mechanism responsible for the variable expression 
TABLE 2 | Advantages and disadvantages of TonB-dependent transporters (TBDTs) in vaccine development.

\begin{tabular}{ll}
\hline Advantages & \multicolumn{1}{c}{ Disadvantages } \\
\hline 1 Surface exposure & 1 Antigenic variation \\
2 Inducible expression during infection & 2 Single TBDT antigen is \\
3 Essential for bacterial virulence & sometimes insufficient for the \\
4 Wide distribution in Gram-negative & vaccine development \\
bacteria & \\
5 Eliciting protective immune responses & \\
6 The DNA sequences of TBDTs have \\
distinctive features, which can be in silico \\
annotated.
\end{tabular}

of some iron transport proteins, including $\mathrm{LbpAB}, \mathrm{HpuAB}$, and FetA. These proteins contain polymeric repeat regions in their encoding loci and promote a rapid on-off switching of protein expression (Bayliss et al., 2001; Harrison et al., 2013).

A single TBDT antigen may be insufficient to protect against bacterial infection because it is unlikely to have $100 \%$ epidemic coverage in the pathogenic strains due to its frequent variation (Sarkissian et al., 2019). Therefore, multiple TBDTs are occasionally required to prepare a qualified vaccine against infection.

Overall, a large number of TBDTs with transporting function of certain rare nutrients have been demonstrated to have application potential in the immune control of infection caused by Gram-negative bacteria. This large category of

\section{REFERENCES}

Abdelhamed, H., Ibrahim, I., Nho, S. W., Banes, M. M., Wills, R. W., Karsi, A., et al. (2017). Evaluation of three recombinant outer membrane proteins, OmpA1, Tdr, and TbpA, as potential vaccine antigens against virulent Aeromonas hydrophila infection in channel catfish (Ictalurus punctatus). Fish Shellfish Immunol. 66, 480-486. doi: 10.1016/j.fsi.2017.05.043

Afonina, G., Leduc, I., Nepluev, I., Jeter, C., Routh, P., Almond, G., et al. (2006). Immunization with the Haemophilus ducreyi hemoglobin receptor HgbA protects against infection in the swine model of chancroid. Infect. Immun. 74 (4), 2224-2232. doi: 10.1128/IAI.74.4.2224-2232.2006

Aghajani, Z., Rasooli, I., and Mousavi Gargari, S. L. (2019). Exploitation of two siderophore receptors, BauA and $\mathrm{BfnH}$, for protection against Acinetobacter baumannii infection. APMIS 127 (12), 753-763. doi: 10.1111/apm.12992

Ala'Aldeen, D. A., Davies, H. A., and Borriello, S. P. (1994). Vaccine potential of meningococcal FrpB: studies on surface exposure and functional attributes of common epitopes. Vaccine 12 (6), 535-541. doi: 10.1016/0264-410x(94)90314-x

Alteri, C. J., Hagan, E. C., Sivick, K. E., Smith, S. N., and Mobley, H. L. (2009). Mucosal immunization with iron receptor antigens protects against urinary tract infection. PloS Pathog. 5 (9), e1000586. doi: 10.1371/journal.ppat.1000586

Bayliss, C. D., Field, D., and Moxon, E. R. (2001). The simple sequence contingency loci of Haemophilus influenzae and Neisseria meningitidis. J. Clin. Invest. 107 (6), 657-662. doi: 10.1172/JCI12557

Becerra, G., Igeno, M. I., Merchan, F., Sanchez-Clemente, R., and Blasco, R. (2020). New evolving strategies revealed by transcriptomic analysis of a fur(-) mutant of the cyanotrophic bacterium Pseudomonas pseudoalcaligenes CECT 5344. Microb. Biotechnol. 13 (1), 148-161. doi: 10.1111/1751-7915.13408

Boulton, I. C., Yost, M. K., Anderson, J. E., and Cornelissen, C. N. (2000). Identification of discrete domains within gonococcal transferrin-binding protein A that are necessary for ligand binding and iron uptake functions. Infect. Immun. 68 (12), 6988-6996. doi: 10.1128/IAI.68.12.6988-6996.2000 molecules will definitely play a non-negligible role in the future vaccine development.

\section{AUTHOR CONTRIBUTIONS}

Conceptualization, YC and WH. Writing-original draft preparation, JW, KX, and QP. Supervision, YC and WH. All authors contributed to the article and approved the submitted version.

\section{FUNDING}

YC was supported by the National Natural Science Foundation of China under Grant number 81974299, the Department of Science and Technology of Sichuan province under Grant number 2019YJ0693, the Southwest Medical University-Traditional Medicine Hospital Affiliated to Southwest Medical University under Grant number 2018XYLH-017, and the Scientific Research Starting Foundation of Traditional Medicine Hospital Affiliated to Southwest Medical University (2019119). JW was supported by the Southwest Medical University-Traditional Medicine Hospital Affiliated to Southwest Medical University under Grant number 2020XYLH-078. KX was supported by the National Natural Science Foundation of China under Grant number 31500116.

Brumbaugh, A. R., Smith, S. N., and Mobley, H. L. (2013). Immunization with the yersiniabactin receptor, FyuA, protects against pyelonephritis in a murine model of urinary tract infection. Infect. Immun. 81 (9), 3309-3316. doi: 10.1128/IAI.00470-13

Cornelissen, C. N., Anderson, J. E., Boulton, I. C., and Sparling, P. F. (2000). Antigenic and sequence diversity in gonococcal transferrin-binding protein A. Infect. Immun. 68 (8), 4725-4735. doi: 10.1128/IAI.68.8.4725-4735.2000

Cornelissen, C. N. (2008). Identification and characterization of gonococcal iron transport systems as potential vaccine antigens. Future Microbiol. 3 (3), 287 298. doi: 10.2217/17460913.3.3.287

Criss, A. K., Kline, K. A., and Seifert, H. S. (2005). The frequency and rate of pilin antigenic variation in Neisseria gonorrhoeae. Mol. Microbiol. 58 (2), 510-519. doi: 10.1111/j.1365-2958.2005.04838.x

del Rio, M. L., Navas, J., Martin, A. J., Gutierrez, C. B., Rodriguez-Barbosa, J. I., and Rodriguez Ferri, E. F. (2006). Molecular characterization of Haemophilus parasuis ferric hydroxamate uptake $(f h u)$ genes and constitutive expression of the FhuA receptor. Vet. Res. 37 (1), 49-59. doi: 10.1051/vetres:2005039

Eijkelkamp, B. A., Hassan, K. A., Paulsen, I. T., and Brown, M. H. (2011). Investigation of the human pathogen Acinetobacter baumannii under iron limiting conditions. BMC Genomics 12, 126. doi: 10.1186/1471-2164-12-126

Frohlich, K. S., Forstner, K. U., and Gitai, Z. (2018). Post-transcriptional gene regulation by an Hfq-independent small RNA in Caulobacter crescentus. Nucleic Acids Res. 46 (20), 10969-10982. doi: 10.1093/nar/gky765

Garenaux, A., Caza, M., and Dozois, C. M. (2011). The Ins and Outs of siderophore mediated iron uptake by extra-intestinal pathogenic Escherichia coli. Vet. Microbiol. 153 (1-2), 89-98. doi: 10.1016/j.vetmic.2011.05.023

Goel, V. K., and Kapil, A. (2001). Monoclonal antibodies against the iron regulated outer membrane Proteins of Acinetobacter baumannii are bactericidal. BMC Microbiol. 1, 16. doi: 10.1186/1471-2180-1-16

Gorden, P. J., Kleinhenz, M. D., Ydstie, J. A., Brick, T. A., Slinden, L. M., Peterson, M. P., et al. (2018). Efficacy of vaccination with a Klebsiella pneumoniae siderophore receptor protein vaccine for reduction of Klebsiella mastitis in 
lactating cattle. J. Dairy Sci. 101 (11), 10398-10408. doi: 10.3168/jds.201714267

Grassmann, A. A., Kremer, F. S., Dos Santos, J. C., Souza, J. D., Pinto, L. D. S., and McBride, A. J. A. (2017). Discovery of Novel Leptospirosis Vaccine Candidates Using Reverse and Structural Vaccinology. Front. Immunol. 8, 463. doi: 10.3389/fimmu.2017.00463

Greene, C., Vadlamudi, G., Newton, D., Foxman, B., and Xi, C. (2016). The influence of biofilm formation and multidrug resistance on environmental survival of clinical and environmental isolates of Acinetobacter baumannii. Am. J. Infect. Control 44 (5), e65-e71. doi: 10.1016/j.ajic.2015.12.012

Gresock, M. G., and Postle, K. (2017). Going Outside the TonB Box: Identification of Novel FepA-TonB Interactions In Vivo. J. Bacteriol. 199 (10), e00649-16. doi: 10.1128/JB.00649-16

Guillier, M., and Gottesman, S. (2008). The 5' end of two redundant sRNAs is involved in the regulation of multiple targets, including their own regulator. Nucleic Acids Res. 36 (21), 6781-6794. doi: 10.1093/nar/gkn742

Habibi, M., Asadi Karam, M. R., and Bouzari, S. (2017). Evaluation of prevalence, immunogenicity and efficacy of FyuA iron receptor in uropathogenic Escherichia coli isolates as a vaccine target against urinary tract infection. Microb. Pathog. 110, 477-483. doi: 10.1016/j.micpath.2017.07.037

Hagan, E. C., and Mobley, H. L. (2007). Uropathogenic Escherichia coli outer membrane antigens expressed during urinary tract infection. Infect. Immun. 75 (8), 3941-3949. doi: 10.1128/IAI.00337-07

Harrison, O. B., Bennett, J. S., Derrick, J. P., Maiden, M. C. J., and Bayliss, C. D. (2013). Distribution and diversity of the haemoglobin-haptoglobin ironacquisition systems in pathogenic and non-pathogenic Neisseria. Microbiology 159 (Pt 9), 1920-1930. doi: 10.1099/mic.0.068874-0

Hassan, A., Naz, A., Obaid, A., Paracha, R. Z., Naz, K., Awan, F. M., et al. (2016). Pangenome and immuno-proteomics analysis of Acinetobacter baumannii strains revealed the core peptide vaccine targets. BMC Genomics 17 (1), 732. doi: 10.1186/s12864-016-2951-4

Hennigar, S. R., and McClung, J. P. (2016). Nutritional Immunity: Starving Pathogens of Trace Minerals. Am. J. Lifestyle Med. 10 (3), 170-173. doi: $10.1177 / 1559827616629117$

Hu, Y., Cong, Y., Li, S., Rao, X., Wang, G., and Hu, F. (2009). Identification of in vivo induced protein antigens of Salmonella enterica serovar Typhi during human infection. Sci. China C Life Sci. 52 (10), 942-948. doi: 10.1007/s11427009-0127-z

Hu, Y. H., Dang, W., and Sun, L. (2012). A TonB-dependent outer membrane receptor of Pseudomonas fluorescens: virulence and vaccine potential. Arch. Microbiol. 194 (9), 795-802. doi: 10.1007/s00203-012-0812-3

Kaneshige, T., Yaguchi, K., and Ohgitani, T. (2009). Siderophore receptor IroN is an important protective antigen against Salmonella infection in chickens. Avian Dis. 53 (4), 563-567. doi: 10.1637/8925-051309-Reg.1

Kelley, L. A., Mezulis, S., Yates, C. M., Wass, M. N., and Sternberg, M. J. (2015). The Phyre2 web portal for protein modeling, prediction and analysis. Nat. Protoc. 10 (6), 845-858. doi: 10.1038/nprot.2015.053

Ketley, J. M. (1997). Pathogenesis of enteric infection by Campylobacter. Microbiology 143 ( Pt 1), 5-21. doi: 10.1099/00221287-143-1-5

Kingsley, R. A., Reissbrodt, R., Rabsch, W., Ketley, J. M., Tsolis, R. M., Everest, P., et al. (1999). Ferrioxamine-mediated Iron(III) utilization by Salmonella enterica. Appl. Environ. Microbiol. 65 (4), 1610-1618. doi: 10.1128/ AEM.65.4.1610-1618.1999

Kortekaas, J., Muller, S. A., Ringler, P., Gregorini, M., Weynants, V. E., Rutten, L., et al. (2006). Immunogenicity and structural characterisation of an in vitro folded meningococcal siderophore receptor (FrpB, FetA). Microbes Infect. 8 (8), 2145-2153. doi: 10.1016/j.micinf.2006.04.011

Krewulak, K. D., and Vogel, H. J. (2011). TonB or not TonB: is that the question? Biochem. Cell Biol. 89 (2), 87-97. doi: 10.1139/o10-141

Kumar, P., Sannigrahi, S., and Tzeng, Y. L. (2012). The Neisseria meningitidis ZnuD zinc receptor contributes to interactions with epithelial cells and supports heme utilization when expressed in Escherichia coli. Infect. Immun. 80 (2), 657-667. doi: 10.1128/IAI.05208-11

Launay, O., Sadorge, C., Jolly, N., Poirier, B., Bechet, S., van der Vliet, D., et al. (2009). Safety and immunogenicity of SC599, an oral live attenuated Shigella dysenteriae type-1 vaccine in healthy volunteers: results of a Phase 2, randomized, double-blind placebo-controlled trial. Vaccine 27 (8), 11841191. doi: 10.1016/j.vaccine.2008.12.021
Leduc, I., Banks, K. E., Fortney, K. R., Patterson, K. B., Billings, S. D., Katz, B. P., et al. (2008). Evaluation of the repertoire of the TonB-dependent receptors of Haemophilus ducreyi for their role in virulence in humans. J. Infect. Dis. 197 (8), 1103-1109. doi: 10.1086/586901

Leduc, I., Fusco, W. G., Choudhary, N., Routh, P. A., Cholon, D. M., Hobbs, M. M., et al. (2011). Passive immunization with a polyclonal antiserum to the hemoglobin receptor of Haemophilus ducreyi confers protection against a homologous challenge in the experimental swine model of chancroid. Infect. Immun. 79 (8), 3168-3177. doi: 10.1128/IAI.00017-11

Lee, J. W., and Helmann, J. D. (2007). Functional specialization within the Fur family of metalloregulators. Biometals 20 (3-4), 485-499. doi: 10.1007/s10534006-9070-7

Liu, M., Huang, M., Shui, Y., Biville, F., Zhu, D., Wang, M., et al. (2018). Roles of B739_1343 in iron acquisition and pathogenesis in Riemerella anatipestifer CH-1 and evaluation of the RA-CH-1DeltaB739_1343 mutant as an attenuated vaccine. PloS One 13 (5), e0197310. doi: 10.1371/journal.pone.0197310

Lu, F., Miao, S., Tu, J., Ni, X., Xing, L., Yu, H., et al. (2013). The role of TonBdependent receptor TbdR1 in Riemerella anatipestifer in iron acquisition and virulence. Vet. Microbiol. 167 (3-4), 713-718. doi: 10.1016/j.vetmic.2013.08.020

Masri, H. P., and Cornelissen, C. N. (2002). Specific ligand binding attributable to individual epitopes of gonococcal transferrin binding protein A. Infect. Immun. 70 (2), 732-740. doi: 10.1128/IAI.70.2.732-740.2002

Mastropasqua, M. C., D’Orazio, M., Cerasi, M., Pacello, F., Gismondi, A., Canini, A., et al. (2017). Growth of Pseudomonas aeruginosa in zinc poor environments is promoted by a nicotianamine-related metallophore. Mol. Microbiol. 106 (4), 543561. doi: $10.1111 / \mathrm{mmi} .13834$

Mellata, M., Touchman, J. W., and Curtiss, R. (2009). Full sequence and comparative analysis of the plasmid pAPEC-1 of avian pathogenic E. coli chi7122 (O78:K80:H9). PloS One 4 (1), e4232. doi: 10.1371/journal.pone. 0004232

Mellata, M., Mitchell, N. M., Schodel, F., Curtiss, R. R., and Pier, G. B. (2016). Novel vaccine antigen combinations elicit protective immune responses against Escherichia coli sepsis. Vaccine 34 (5), 656-662. doi: 10.1016/ j.vaccine.2015.12.014

Mobley, H. L., and Alteri, C. J. (2015). Development of a Vaccine against Escherichia coli Urinary Tract Infections. Pathogens 5 (1), 1-8. doi: 10.3390/ pathogens5010001

Morton, D. J., Seale, T. W., Madore, L. L., VanWagoner, T. M., Whitby, P. W., and Stull, T. L. (2007). The haem-haemopexin utilization gene cluster ( $h x u C B A)$ as a virulence factor of Haemophilus influenzae. Microbiology 153 (Pt 1), 215-224. doi: 10.1099/mic.0.2006/000190-0

Nepluev, I., Afonina, G., Fusco, W. G., Leduc, I., Olsen, B., Temple, B., et al. (2009). An immunogenic, surface-exposed domain of Haemophilus ducreyi outer membrane protein $\mathrm{HgbA}$ is involved in hemoglobin binding. Infect. Immun. 77 (7), 3065-3074. doi: 10.1128/IAI.00034-09

Ni, Z., Chen, Y., Ong, E., and He, Y. (2017). Antibiotic Resistance DeterminantFocused Acinetobacter baumannii Vaccine Designed Using Reverse Vaccinology. Int. J. Mol. Sci. 18 (2), 458. doi: 10.3390/ijms18020458

Nielubowicz, G. R., Smith, S. N., and Mobley, H. L. (2008). Outer membrane antigens of the uropathogen Proteus mirabilis recognized by the humoral response during experimental murine urinary tract infection. Infect. Immun. 76 (9), 4222-4231. doi: 10.1128/IAI.00533-08

Nikaido, H. (2003). Molecular basis of bacterial outer membrane permeability revisited. Microbiol. Mol. Biol. Rev. 67 (4), 593-656. doi: 10.1128/ MMBR.67.4.593-656.2003

Noinaj, N., Guillier, M., Barnard, T. J., and Buchanan, S. K. (2010). TonBdependent transporters: regulation, structure, and function. Annu. Rev. Microbiol. 64, 43-60. doi: 10.1146/annurev.micro.112408.134247

Noinaj, N., Buchanan, S. K., and Cornelissen, C. N. (2012). The transferrin-iron import system from pathogenic Neisseria species. Mol. Microbiol. 86 (2), $246-$ 257. doi: $10.1111 / \mathrm{mmi} .12002$

Noinaj, N., Cornelissen, C. N., and Buchanan, S. K. (2013). Structural insight into the lactoferrin receptors from pathogenic Neisseria. J. Struct. Biol. 184 (1), 8392. doi: 10.1016/j.jsb.2013.02.009

Pettersson, A., Kuipers, B., Pelzer, M., Verhagen, E., Tiesjema, R. H., Tommassen, J., et al. (1990). Monoclonal antibodies against the 70-kilodalton iron-regulated protein of Neisseria meningitidis are bactericidal and strain specific. Infect. Immun. 58 (9), 3036-3041. doi: 10.1128/IAI.58.9.3036-3041.1990 
Pienko, T., and Trylska, J. (2020). Extracellular loops of BtuB facilitate transport of vitamin B12 through the outer membrane of E. coli. PloS Comput. Biol. 16 (7), e1008024. doi: 10.1371/journal.pcbi.1008024

Russo, T. A., McFadden, C. D., Carlino-MacDonald, U. B., Beanan, J. M., Olson, R., and Wilding, G. E. (2003). The Siderophore receptor IroN of extraintestinal pathogenic Escherichia coli is a potential vaccine candidate. Infect. Immun. 71 (12), 7164-7169. doi: 10.1128/IAI.71.12.7164-7169.2003

Sadorge, C., Ndiaye, A., Beveridge, N., Frazer, S., Giemza, R., Jolly, N., et al. (2008). Phase 1 clinical trial of live attenuated Shigella dysenteriae type-1 DeltaicsA Deltaent Deltafep DeltastxA:HgR oral vaccine SC599 in healthy human adult volunteers. Vaccine 26 (7), 978-987. doi: 10.1016/j.vaccine.2007.11.024

Saleem, M., Prince, S. M., Patel, H., Chan, H., Feavers, I. M., and Derrick, J. P. (2012). Refolding, purification and crystallization of the FrpB outer membrane iron transporter from Neisseria meningitidis. Acta Crystallogr. Sect. F Struct. Biol. Cryst. Commun. 68 (Pt 2), 231-235. doi: 10.1107/S1744309111056028

Sarkissian, C. A., Alteri, C. J., and Mobley, H. L. T. (2019). UTI patients have preexisting antigen-specific antibody titers against UTI vaccine antigens. Vaccine 37 (35), 4937-4946. doi: 10.1016/j.vaccine.2019.07.031

Schalk, I. J., Mislin, G. L., and Brillet, K. (2012). Structure, function and binding selectivity and stereoselectivity of siderophore-iron outer membrane transporters. Curr. Top. Membr. 69, 37-66. doi: 10.1016/B978-0-12-3943903.00002-1

Schauer, K., Rodionov, D. A., and de Reuse, H. (2008). New substrates for TonBdependent transport: do we only see the 'tip of the iceberg'? Trends Biochem. Sci. 33 (7), 330-338. doi: 10.1016/j.tibs.2008.04.012

Sen, B., Meeker, A., and Ramakrishnan, G. (2010). The fslE homolog, FTL_0439 $(f u p A / B)$, mediates siderophore-dependent iron uptake in Francisella tularensis LVS. Infect. Immun. 78 (10), 4276-4285. doi: 10.1128/IAI.00503-10

Sen-Kilic, E., Blackwood, C. B., Boehm, D. T., Witt, W. T., Malkowski, A. C., Bevere, J. R., et al. (2019). Intranasal Peptide-Based FpvA-KLH Conjugate Vaccine Protects Mice From Pseudomonas aeruginosa Acute Murine Pneumonia. Front. Immunol. 10, 2497. doi: 10.3389/fimmu.2019.02497

Serino, L., Moriel, D. G., Rappuoli, R., and Pizza, M. (2010). Towards a vaccine against Escherichia coli-associated urinary tract infections. Future Microbiol. 5 (3), 351-354. doi: 10.2217/fmb.10.6

Shivachandra, S. B., Yogisharadhya, R., Kumar, A., Mohanty, N. N., and Nagaleekar, V. K. (2015). Recombinant transferrin binding protein A (rTbpA) fragments of Pasteurella multocida serogroup B:2 provide variable protection following homologous challenge in mouse model. Res. Vet. Sci. 98, 1-6. doi: 10.1016/j.rvsc.2014.11.013

Solis, N., and Cordwell, S. J. (2011). Current methodologies for proteomics of bacterial surface-exposed and cell envelope proteins. Proteomics 11 (15), 31693189. doi: $10.1002 /$ pmic. 201000808

Stork, M., Bos, M. P., Jongerius, I., de Kok, N., Schilders, I., Weynants, V. E., et al. (2010). An outer membrane receptor of Neisseria meningitidis involved in zinc acquisition with vaccine potential. PloS Pathog. 6, e1000969. doi: 10.1371/ journal.ppat.1000969

Stork, M., Grijpstra, J., Bos, M. P., Manas Torres, C., Devos, N., Poolman, J. T., et al. (2013). Zinc piracy as a mechanism of Neisseria meningitidis for evasion of nutritional immunity. PloS Pathog. 9 (10), e1003733. doi: 10.1371/ journal.ppat.1003733

Sun, Y. Y., and Sun, L. (2015). Pseudomonas fluorescens: iron-responsive proteins and their involvement in host infection. Vet. Microbiol. 176 (3-4), 309-320. doi: 10.1016/j.vetmic.2015.01.020

Thompson, E. A. L., Feavers, I. M., and Maiden, M. C. J. (2003). Antigenic diversity of meningococcal enterobactin receptor FetA, a vaccine component. Microbiology 149 (Pt 7), 1849-1858. doi: 10.1099/mic.0.26131-0
Wandersman, C., and Delepelaire, P. (2004). Bacterial iron sources: from siderophores to hemophores. Annu. Rev. Microbiol. 58, 611-647. doi: 10.1146/annurev.micro.58.030603.123811

Wellawa, D. H., Allan, B., White, A. P., and Koster, W. (2020). Iron-Uptake Systems of Chicken-Associated Salmonella Serovars and Their Role in Colonizing the Avian Host. Microorganisms 8 (8), 1203. doi: 10.3390/ microorganisms 8081203

Wen, Y., Yan, X., Wen, Y., Cao, S., He, L., Ding, L., et al. (2016). Immunogenicity of the recombinant HxuCBA proteins encoded by $h x u C B A$ gene cluster of Haemophilus parasuis in mice. Gene 591 (2), 478-483. doi: 10.1016/ j.gene.2016.07.001

West, D., Reddin, K., Matheson, M., Heath, R., Funnell, S., Hudson, M., et al. (2001). Recombinant Neisseria meningitidis transferrin binding protein A protects against experimental meningococcal infection. Infect. Immun. 69 (3), 1561-1567. doi: 10.1128/IAI.69.3.1561-1567.2001

Wieser, A., Romann, E., Magistro, G., Hoffmann, C., Norenberg, D., Weinert, K., et al. (2010). A multiepitope subunit vaccine conveys protection against extraintestinal pathogenic Escherichia coli in mice. Infect. Immun. 78 (8), 3432-3442. doi: 10.1128/IAI.00174-10

Williams, P. H., Rabsch, W., Methner, U., Voigt, W., Tschape, H., and Reissbrodt, R. (2006). Catecholate receptor proteins in Salmonella enterica: role in virulence and implications for vaccine development. Vaccine 24 (18), 3840-3844. doi: 10.1016/j.vaccine.2005.07.020

Xiong, K., Chen, Z., Xiang, G., Wang, J., Rao, X., Hu, F., et al. (2012). Deletion of $y n c D$ gene in Salmonella enterica ssp. enterica serovar Typhi leads to attenuation in mouse model. FEMS Microbiol. Lett. 328 (1), 70-77. doi: 10.1111/j.1574-6968.2011.02481.x

Xiong, K., Chen, Z., Zhu, C., Li, J., Hu, X., Rao, X., et al. (2015). Safety and immunogenicity of an attenuated Salmonella enterica serovar Paratyphi A vaccine candidate. Int. J. Med. Microbiol. 305 (6), 563-571. doi: 10.1016/ j.ijmm.2015.07.004

Zeng, X., Xu, F., and Lin, J. (2009). Molecular, antigenic, and functional characteristics of ferric enterobactin receptor CfrA in Campylobacter jejuni. Infect. Immun. 77 (12), 5437-5448. doi: 10.1128/IAI.00666-09

Zeng, L., Wang, D., Hu, N., Zhu, Q., Chen, K., Dong, K., et al. (2017). A Novel Pan-Genome Reverse Vaccinology Approach Employing a Negative-Selection Strategy for Screening Surface-Exposed Antigens against leptospirosis. Front. Microbiol. 8, 396. doi: 10.3389/fmicb.2017.00396

Zhang, Y., Teper, D., Xu, J., and Wang, N. (2019). Stringent response regulators (p)ppGpp and DksA positively regulate virulence and host adaptation of Xanthomonas citri. Mol. Plant Pathol. 20 (11), 1550-1565. doi: 10.1111/ mpp. 12865

Zhu, C., Xiong, K., Chen, Z., Hu, X., Li, J., Wang, Y., et al. (2015). Construction of an attenuated Salmonella enterica serovar Paratyphi A vaccine strain harboring defined mutations in $h t r A$ and $y n c D$. Microbiol. Immunol. 59 (8), 443-451. doi: 10.1111/1348-0421.12276

Conflict of Interest: The authors declare that the research was conducted in the absence of any commercial or financial relationships that could be construed as a potential conflict of interest.

Copyright $\odot 2021$ Wang, Xiong, Pan, He and Cong. This is an open-access article distributed under the terms of the Creative Commons Attribution License (CC BY). The use, distribution or reproduction in other forums is permitted, provided the original author(s) and the copyright owner(s) are credited and that the original publication in this journal is cited, in accordance with accepted academic practice. No use, distribution or reproduction is permitted which does not comply with these terms. 